\title{
One Model of a Linear-Fractional Three-Index Transportation Problem
}

\author{
Prof. PhD Rosen Nikolaev \\ University of Economics - Varna, Varna, Bulgaria \\ nikolaev_rosen@ue-varna.bg \\ Assoc. Prof. PhD Tanka Milkova \\ University of Economics - Varna, Varna, Bulgaria \\ tankamilkova@ue-varna.bg
}

\begin{abstract}
In specialized literature and in practice some methods for production-transportation planning using the models of three-index transportation problem are well-known. Different linear-fractional models for productiontransportation planning are applied as well. In the present paper the authors aim to combine elements of the threeindex transportation problem and elements of the linear-fractional optimization, thus offering a model of three-index transportation problem with a linear-fractional function which combines the advantages of both methods and gives better opportunities for determining an optimal solution and making the post-optimal analysis.
\end{abstract}

Keywords: three-index transportation problem, linear-fractional programming

JEL Code: C61 DOI: https://doi.org/10.36997/IJUSV-ESS/2020.9.1.129

\section{Въведение}

В съвременните условия на силно динамична, нестабилна и високо конкурентна среда пред всички участници на пазара е поставено предизвикателството непрекъснато и последователно да търсят възможности за осигуряване на необходимата икономическа ефективност, в резултат на което да отстояват конкурентните си предимства. Върху утвърждаването и разрастването на пазарните позиции на всеки стопански субект оказват влияние редица фактори, но един от тях може да се сведе до осъществяването на оптимално планиране на производствено-транспортните дейности на предприятието. Концепцията за разглеждане на предприятието като система, а не като съвкупност от отделни елементи се свързва с постигане на по-добри управленски резултати в различни ключови направления. Това обстоятелство предопределя използването на научния апарат на системния анализ и един от най-ефективните му методи - икономико-математическото моделиране на икономически системи. В предишни свои публикации авторите са изследвали възможностите за оптимално планиране на производствено-транспортните дейности на предприятието с относителни критерии, чрез позоваване на методите на дробно-линейното (хиперболичното) оптимиране (Николаев, 2016). Едно от най-силните предимства на дробнолинейното оптимиране се състои в съществуващата възможност за извършване на оптимизация по два критерия, което в по-голяма степен отчита специфичните особености на конкретната ситуация. Също така са изследвани възможностите за оптимизиране на производствено-транспортните дейности на предприятието с помощта на различни модификации на транспортна задача. Конструирани са редица модели, които обхващат покомплексно проблеми на производствено-транспортните дейности на база трииндексна линейна транспортна задача.

В настоящата разработка авторите си поставят за цел да съчетаят елементи на трииндексната транспортна задача и елементи на дробно-линейното оптимиране и да предложат модел на трииндексна транспортна задача с дробно-линейна целева функция, която съчетава предимствата от двата метода и предоставя по-широки възможности за осигуряване на оптимално решение и извършване на следоптимален анализ. 


\section{1. Математически основи на трииндексната транспортна задача} 2019).

Трииндексната транспортна задача се дефинира по следния начин (Николаев и др.,

Трябва да се намери минимумът на линейната функция

$$
Z(X)=\sum_{i=1}^{m} \sum_{s=1}^{k} \sum_{j=1}^{n} c_{i s j} x_{i s j}
$$

при следните ограничителни условия

$$
\begin{aligned}
& \sum_{s=1}^{k} \sum_{i=1}^{m} x_{i s j}=b_{j},(j=1 \div n), \\
& \sum_{i=1}^{m} \sum_{j=1}^{n} x_{i s j}=d_{s},(s=1 \div k), \\
& \sum_{j=1}^{n} \sum_{s=1}^{k} x_{i s j}=a_{i},(i=1 \div m), \\
& x_{i s j} \geq 0,(i=1 \div m, s=1 \div k, j=1 \div n) .
\end{aligned}
$$

Предполага се, че са изпълнени балансиращите условия

$$
\sum_{i=1}^{m} a_{i}=\sum_{s=1}^{k} d_{s}=\sum_{j=1}^{n} b_{j}=M^{2}
$$

Твърдение. необходимото и достатъчно условие трииндексната транспортна задача да има решение е да бъдат изпълнени балансиращите условия (6).

Доказателство. Необходимост: нека трииндексната транспортна задача (1) - (.6) да има решение $X=\left\{x_{i s j}\right\}$. Това означава, че $X=\left\{x_{i s j}\right\}$ удовлетворява ограничителните условия $(2)-(5)$ :

$$
\begin{aligned}
& \sum_{s=1}^{k} \sum_{i=1}^{m} x_{i s j}=b_{j},(j=1 \div n), \\
& \sum_{i=1}^{m} \sum_{j=1}^{n} x_{i s j}=d_{s},(s=1 \div k), \\
& \sum_{j=1}^{n} \sum_{s=1}^{k} x_{i s j}=a_{i},(i=1 \div m) .
\end{aligned}
$$

Сумират се всички равенства (2') по индекса $j$ и се получава $\sum_{j=1}^{n} \sum_{s=1}^{k} \sum_{i=1}^{m}=\sum_{j=1}^{n} b_{j}$. Аналогично се сумират всички равенства (3') и (4') съответно по индексите $s$ и $i$ и се намира

$$
\sum_{s=1}^{k} \sum_{i=1}^{m} \sum_{j=1}^{n} x_{i s j}=\sum_{s=1}^{k} d_{s}, \sum_{i=1}^{m} \sum_{j=1}^{n} \sum_{s=1}^{k} x_{i s j}=\sum_{i=1}^{m} a_{i} .
$$

Левите страни на всички равенства представят едни и същи суми, следователно и десните им страни също са равни, т.е. изпълнено е балансиращото условие (6).

Достатъчност. Нека предположим, че е изпълнено балансиращото условие (6)

$$
\sum_{i=1}^{m} a_{i}=\sum_{s=1}^{k} d_{s}=\sum_{j=1}^{n} b_{j}=M^{2} .
$$

Числата $\bar{x}_{i s j}=\frac{a_{i} d_{s} b_{j}}{M^{2}} \geq 0(i=1 \div m, s=1 \div k, j=1 \div n)$ са решение на трииндексната транспортна задача, тъй като удовлетворяват всички нейни ограничителни условия 


$$
\begin{aligned}
& \sum_{s=1}^{k} \sum_{i=1}^{m} \bar{x}_{i s j}=\sum_{s=1}^{k} \sum_{i=1}^{m} \frac{a_{i} d_{s} b_{j}}{M^{2}}=\frac{b_{j}}{M^{2}} \sum_{s=1}^{k}\left(\sum_{i=1}^{m} a_{i} d_{s}\right)= \\
& =\frac{b_{j}}{M^{2}} \sum_{s=1}^{k} d_{s} \sum_{i=1}^{m} a_{i}=\frac{b_{j}}{M^{2}} \cdot M \cdot M=b_{j}, \quad j=1 \div n ; \\
& \sum_{i=1}^{m} \sum_{j=1}^{n} \bar{x}_{i s j}=\sum_{i=1}^{m} \sum_{j=1}^{n} \frac{a_{i} d_{s} b_{j}}{M^{2}}=\frac{d_{s}}{M^{2}} \sum_{i=1}^{m}\left(\sum_{j=1}^{n} a_{i} b_{j}\right)= \\
& =\frac{d_{s}}{M^{2}} \sum_{i=1}^{m} a_{i} \sum_{j=}^{n} b_{j}=\frac{d_{s}}{M^{2}} \cdot M \cdot M=d_{s}, \quad s=1 \div k ; \\
& \sum_{j=1}^{n} \sum_{s=1}^{k} \bar{x}_{i s j}=\sum_{j=1}^{n} \sum_{s=1}^{k} \frac{a_{i} d_{s} b_{j}}{M^{2}}=\frac{a_{i}}{M^{2}} \sum_{j=1}^{n}\left(\sum_{s=1}^{k} d_{s} b_{j}\right)= \\
& =\frac{a_{i}}{M^{2}} \sum_{j=1}^{n} b_{j} \sum_{s=1}^{k} d_{s}=\frac{a_{i}}{M^{2}} \cdot M \cdot M=a_{i}, i=1 \div m .
\end{aligned}
$$

Следователно множеството от допустимите решения на трииндексната транспортна задача не е празно. Нещо повече, това множество е ограничено, тъй като възможните стойности на всяка от променливите $x_{i s j}$ са в интервала $\left[0, \min \left(a_{i}, d_{s}, b_{j}\right)\right]$. Следователно многостенното множество $K$, определено от ограничителните условия (2) - (6) е ограничено. Освен това целевата функция (1) е непрекъсната (като линейна функция), затова достига в множеството от допустимите решения своята долна граница. Следователно за трииндексната транспортна задача при условие (6) може да се твърди, че винаги има оптимално решение.

\section{2. Теоретични основи на дробно-линейното оптимиране}

Както е известно задачата на дробно-линейното (хиперболичното) оптимиране, т.е. задачата за оптимизацията на частното от две линейни форми при линейни ограничителни условия, има реален икономически смисъл. Като типичен пример за такова съдържание е достигането на минимална себестойност при производството на някакъв продукт (или осъществяване на някакви превози). По-конкретно това може да се демонстрира с възможността за минимизиране себестойността (тук се предполага, че обемът на произвеждания продукт (или осъществявани превози) не е предварително фиксиран, тъй като тогава, минималната себестойност се достига при минимални разходи (Иванов и др., 2000). И при такава ситуация могат да се използват методите на линейното оптимиране) на производството на някакъв еднороден продукт, който може да се изготвя по $R_{j}(j=1 \div n)$ технологични начини. Ако $c_{j}$ представлява интензивността на $R_{j}$-тия начин, a $d_{j}-$ съответните разходи за единица време, то при стратегия $X=\left(x_{1}, x_{2}, \ldots, x_{n}\right)$ (единиците време за всеки от начините $R_{j}$ ) себестойността на произвеждания продукт ще се определя посредством функцията

$$
Z(X)=\frac{\sum_{j=1}^{n} c_{j} x_{j}+c_{0}}{\sum_{j=1}^{n} d_{j} x_{j}+d_{0}}(\text { в лева за единица продукт), }
$$

т.е. тя се изразява като отношение на две линейни форми (тук $c_{0}$ и $d_{0}$ са параметри, независещи от интензивностите $\left.x_{j}(j=1 \div n)\right)$. 
Аналогично, ако става въпрос за разпределение на $m$ превозни средства по $n$ различни маршрути, то при производителност $c_{i j}$ (тонкилометри за единица време) на $i$-тото превозно средство по $j$-тия маршрут и съответните експлоатационни разходи $d_{i j}$ (в лева за единица време) ще се достигне до това, че себестойността на превозите при план $X=\left\|x_{i j}\right\|$ (единиците време на разпределение на $i$-тото превозно средство по $j$-тия маршрут) се определя посредством отношението

$$
Z(X)=\frac{\sum_{i=1}^{m} \sum_{j=1}^{n} c_{i j} x_{i j}}{\sum_{i=1}^{m} \sum_{j=1}^{n} d_{i j} x_{i j}} \text { (в лева за тонкилометри). }
$$

Като други примери могат да бъдат посочени икономико-математически модели за максимална рентабилност и въобще оптимизационни задачи с относителни критерии за оптималност (трудоемкост, производителност, материалоемкост, капиталоотдаване и т.н.). Задачата на хиперболичното оптимиране твърде отдавна намира също приложение в теорията на игрите (Charnes et al., 1962), в мрежовите транспортни задачи (Martos, 1961) и др.

Хиперболичното оптимиране е предмет на изследване в редица разработки, където самата задача се свежда до две спомагателни задачи на линейното оптимиране (Сухаров и др., 1986), като при това най-често се изключва случаят на неограничена (безкрайна) област, определена от ограничителните условия. Някои автори (Кендеров и др., 1989) също свеждат задачата на хиперболичното оптимиране до спомагателна (една задача) на линейното оптимиране, като от нейното решение се намира това на самата изходна задача.

Разглеждаме задачата на хиперболичното оптимиране във вида:

$$
\max (\min ): Z(X)=\frac{\sum_{j=1}^{n} c_{j} x_{j}}{\sum_{j=1}^{n} d_{j} x_{j}}=\frac{Z_{1}(X)}{Z_{2}(X)}
$$

при линейни ограничения

$$
\begin{aligned}
& \sum_{j=1}^{n} a_{i j} x_{j}=b_{i},(i=1 \div m), \\
& x_{j} \geq 0,(j=1 \div n) .
\end{aligned}
$$

Тази задача се възприема като стандартна задача на хиперболичното оптимиране и всеки друг вид на нейния запис може да бъде сведен до тази стандартна форма. Така например критерият (7) лесно може да придобие вида (8) посредством полагането

$$
x_{j}=y_{j}+x_{j}^{0} .
$$

Като се заместят тези значения на променливите в (7), с цел да се достигне до еднороден характер на целевата функция, трябва да са в сила равенствата:

$$
\mid \begin{aligned}
& \sum_{j=1}^{n} c_{j} x_{j}^{0}+c_{0}=0 \\
& \sum_{j=1}^{n} d_{j} x_{j}^{0}+d_{0}=0
\end{aligned}
$$

Както и при задачата на линейното оптимиране множеството от допустимите решения (9) - (10) е изпъкнало, имащо краен брой върхови точки (Атанасов и др., 2012). Като се вземе предвид, че знаменателят $Z_{2}(X)$ на целевата функция (8) е линейна, а следователно и 
непрекъсната функция, може за удобство да се приеме, че $Z_{2}(X)>0$ в областта $U$. Изискването $Z_{2}(X)>0$ може да се приеме като допълнително ограничение и в този случай множеството $U$ се определя от (9) - (10) и $Z_{2}(X)>0$. Например на практика е в сила $d_{j} \geq 0$ $(j=1 \div n)$ и условието $Z_{2}(X)>0$ е изпълнено, ако $U$ не съдържа нулевия вектор. В противен случай знакът минус може да бъде отчетен в числителя.

За хиперболичната задача по аналогия на линейното оптимиране се записват основните понятия (допустимо решение, базисно решение, оптимално решение), както и твърденията свързани с тях.

Определение. Хиперболичната задача (8) - (9) е разрешима (има решение) точно тогава, когато съществува вектор $X^{*} \in U$, за който

$$
Z\left(X^{*}\right)=\sup (\inf ) Z(X): X \in U \text {. }
$$

Тук е употребено $\sup (\inf )$ вместо $\max (\min )$, тъй като за разлика от линейната функция хиперболичната функция може да не достига екстремума си върху областта $U$, дори когато последната е ограничена.

За потребностите на практиката е възможно да се избере значително опростен вариант на задачата на хиперболичното оптимиране, който се свежда до следното:

Да се намери максимумът на функцията

$$
F(X)=\frac{\sum_{j=1}^{n} r_{j} x_{j}}{\sum_{j=1}^{n} x_{j}}
$$

при ограничителни условия

$$
\begin{aligned}
& \sum_{j=1}^{n} a_{i j} x_{j} \leq b_{i},(i=1 \div m), \\
& d_{j} \leq x_{j},(j=1 \div n) .
\end{aligned}
$$

Параметрите на модела (11) - (13) са:

$r_{j}$ - рентабилност на $j$-тия вид продукт;

$b_{i}$ - наличност на $i$-тия вид лимитиращи ресурси;

$d_{j}$ - максимално необходимия обем на производство от $j$-тия вид продукт;

$a_{i j}$ - разходни норми от $i$-тия вид ресурси за единица от $j$-тия вид продукт.

С $x_{j}$ са означени неизвестните обеми продукт от $j$-тия вид.

\section{3. Модел на трииндексна транспортна задача с дробно линейна целева функция}

На база теоретичните основи на трииндексната транспортна задача и дробнолинейното оптимиране, може да бъде конструиран следният модел на трииндексна транспортна задача с дробно-линейна целева функция.

Да се намери минимумът на дробно-линейната функция

$$
Z(X)=\frac{\sum_{i=1}^{m} \sum_{s=1}^{k} \sum_{j=1}^{n} c_{i s j} x_{i s j}+c_{0}}{\sum_{i=1}^{m} \sum_{s=1}^{k} \sum_{j=1}^{n} d_{i s j} x_{i s j}+d_{0}}
$$

при следните ограничителни условия 


$$
\begin{aligned}
& \sum_{s=1}^{k} \sum_{i=1}^{m} x_{i s j}=b_{j},(j=1 \div n), \\
& \sum_{i=1}^{m} \sum_{j=1}^{n} x_{i s j}=d_{s},(s=1 \div k), \\
& \sum_{j=1}^{n} \sum_{s=1}^{k} x_{i s j}=a_{i},(i=1 \div m), \\
& x_{i s j} \geq 0,(i=1 \div m, s=1 \div k, j=1 \div n) .
\end{aligned}
$$

Предполага се, че са изпьлнени балансиращите условия

$$
\sum_{i=1}^{m} a_{i}=\sum_{s=1}^{k} d_{s}=\sum_{j=1}^{n} b_{j}=M^{2} .
$$

Конструираният модел е разрешим при условие, че са изпълнени балансиращите условия. Доказателството на това твърдение се препокрива с доказателството на твърдението за разрешимостта на трииндексната транспортна задача с линейна целева функция. За целевата функция важи същото, тя е непрекъсната като дробно-линейна функция с положителен знаменател. Следователно за трииндексната транспортна задача с дробнолинейна целева функция, при условие (19) може да се твърди, че винаги има оптимално решение.

Опростен вариант на модела на трииндексната транспортна задача с дробно-линейна целева функция може да се представи по следния начин:

Да се намери минимумът на дробно-линейната функция

$$
Z(X)=\frac{\sum_{i=1}^{m} \sum_{s=1}^{k} \sum_{j=1}^{n} r_{i s j} x_{i s j}}{\sum_{i=1}^{m} \sum_{s=1}^{k} \sum_{j=1}^{n} x_{i s j}}
$$

при следните ограничителни условия

$$
\begin{aligned}
& \sum_{s=1}^{k} \sum_{i=1}^{m} x_{i s j}=b_{j},(j=1 \div n), \\
& \sum_{i=1}^{m} \sum_{j=1}^{n} x_{i s j}=d_{s},(s=1 \div k), \\
& \sum_{j=1}^{n} \sum_{s=1}^{k} x_{i s j}=a_{i},(i=1 \div m), \\
& x_{i s j} \geq 0,(i=1 \div m, s=1 \div k, j=1 \div n),
\end{aligned}
$$

където $r_{i s j}$ е рентабилността при осъществяване на дейността по $i s j$-ти вариант.

На база теорията на хиперболичното опримиране тук може да се направи следната обосновка за значението на параметрите $c_{i s j}, c_{0}, d_{i s j}, d_{0}$ :

- $\quad c_{i s j}$ - интензивност (производителност) при осъществяване на дейността по $i s j$-ти вариант;

- $c_{0}$ - интензивност (производителност) при осъществяване на дейността независеща от $i s j$-ти вариант;

- $\quad d_{i s j}$ - разходи при осъществяване на дейността по $i s j$-ти вариант;

- $\quad d_{0}$ - разходи при осъществяване на дейността независещи от $i s j$-ти вариант. 
Смисъльт на трите измерения в трииндексната транспортна задача може да бъде различен, съобразно потребностите на конкретната икономическа ситуация. Тук ще предложим следните примерни практически ориентирани интерпретации на трииндексната транспортна задача:

1) В логистичната система трябва да се организира предвижването на еднороден материален поток между доставчици и потребители в продължение на определен период от време. Нека $m$ на брой доставчици $A_{i}(i=1,2, \ldots, m)$ осъществяват снабдяване на $n$ на брой потребители $B_{j}(j=1,2, \ldots, n)$ за определен период от време $T$, включващ $k$ на брой дни $(k=1,2, \ldots, s)$ с определен еднороден продукт. Известно е общото налично количество от продукта с което разполага всеки доставчик за целия период от време, означено с $a_{i}(i=1,2, \ldots, m)$. Известно е също така заявеното от всеки от потребителите количество от продукта, което трябва да им се достави в продължение на целия период от време, означено с $b_{j}(j=1,2, \ldots, n)$. Известни са транспортните разходи $c_{i s j}$ за превоз на една единица от еднородния товар от $i$-ти доставчик до $j$-ти потребител през $s$-ти ден от периода. Основният фактор, оказваш влияние на стойността на транспортните разходи от всеки от доставчиците до всеки от потребителите е разстоянието между тях. Тук се допуска възможността тези разходи да зависят и от деня, в който се осъществява доставката, тъй като в рамките на определен времеви период (седмица, месец, година) интензитетьт на материалните потоци може да гравитира в широки граници под влияние на събития със сезонен, икономически, политически и прочие характер. $\mathrm{C} \quad x_{i s j}$ $(i=1,2, \ldots, m ; j=1,2, \ldots, n ; s=1,2, \ldots, k)$ се означава неизвестното количеството от еднородния товар, което се доставя от $i$-ти доставчик до $j$-ти потребител през $s$-ти ден от периода (Nikolaev et al., 2018; Николаев и др., 2018).

2) В логистичната система трябва да се организира предвижването на разнороден материален поток между доставчици и потребители. Нека $m$ на брой доставчици $A_{i}(i=1,2, \ldots, m)$ осъществяват снабдяване на $n$ на брой потребители $B_{j}(j=1,2, \ldots, n)$ за определен период от време $T$. Трябва да бъдат транспортирани $k$ на брой продукта $(k=1,2, \ldots, s)$ от доставчиците до потребителите. Известно е общото налично количество от всеки от видовете продукти с което разполага всеки доставчик. Известно е също така заявеното от всеки от потребителите количество от всеки от продуктите, което трябва да им се достави. Известни са транспортните разходи $c_{i s j}$ за превоз на една единица от $s$-ти вид продукт от $i$-ти доставчик до $j$-ти потребител. С $x_{i s j}(i=1,2, \ldots, m ; j=1,2, \ldots, n ; s=1,2, \ldots, k)$ се означава неизвестното количеството от $s$-ти вид продукт, което се доставя от $i$-ти доставчик до $j$-ти потребител.

\section{Заключение}

Приложението на моделите на трииндексна производствено-транспортна задача спомага за постигане на оптимални резултати от дейността при отчитане на три показателя едновременно. Основното преимущество на моделите на дробно-линейното оптимиране считаме, че се състои в безспорното им практико-приложно значение и категорично доказаната им възможност да доведат до вземане на оптимални решения при планиране на производствено-транспортните дейности в предприятието при прилагане на два критерия за оптималност. Приложението на двата подхода в съчетание при осъществяване планирането на производствено-транспортните дейности на предприятието води до увеличаване на икономическия ефект от функционирането на организациите, осигурява им сериозни конкурентни предимства и затова следва да бъде приоритет на рационално 
функциониращите стопански субекти. За постигането на тези резултати е необходимо да се търсят оптимални управленски решения, а една от възможностите за определянето им при планиране на дейностите в предприятието е свързана с прилагането на методите на икономико-математическото моделиране.

\section{References}

1. Atanasov, B., R. Nikolaev, R. Miryanov (2012). Kolichestveni metodi v upravlenieto. Varna: Nauka i ikonomika, s. $120-121$.

2. Charnes, A., W. Cooper. (1962) Programming with Linear Fractional Functionals. Naval Res. Logist. Quart. vol. 9, № 3-4.

3. Ivanov, I., Bl. Blagoev. (2000) Ikonomika na predpriyatieto. Varna: Nauka i ikonomika, s. 158.

4. Kenderov, P., G. Hristov, As. Donchev. (1989) Matematichesko optimirane. Sofiya: Universitetsko izdatelstvo „Kliment Ohridski”, s. 146.

5. Martos, B. (1961) Hyperbolic by Simplex Method. 2-eme congr. math. Hon-grois, Budapest.

6. Nikolaev, R., D. Zhelyazkova, T. Milkova, R. Miryanov, V. Yordanova. (2019) Optimizirane na ikonomicheski protsesi $v$ proizvodstveno-transportnata sistema na predpriyatieto. Bibl. „Prof. Tsani Kalyandzhiev”, Kniga shestdeset i vtora. Varna: Nauka i ikonomika.

7. Nikolaev, R., D. Zhelyazkova, T. Milkova. (2018) Minimizing the transportation costs by means of three dimensional transportation problem. // 5rd International multidisciplinary scientific conference on social sciences \& arts SGEM 2018, 26 August - 01 September 2018 Albena, Bulgaria. Conference proceedings - Issue 1.4, Modern science, Economics and Tourism, Volume 5, pp. 549 - 556. DOI: 10.5593/sgemsocial2018/1.4;

8. Nikolaev, R., T. Milkova. (2018) Optimizirane na transportnite razhodi chrez mnogoproduktova transportna zadacha. // Sbornik s dokladi ot mezhdunarodna nauchna konferentsiya „Targoviya 4.0 - nauka, praktika i obrazovanie“. Varna: Nauka i ikonomika, 2018, s. $316-324$.

9. Nikolaev, R. (2016) Optimizirane proizvodstveno-transportnoto planirane na predpriyatieto $s$ otnositelni kriterii. „Prof. Tsani Kalyandzhiev”, Varna: Nauka i ikonomika.

10. Suharov, A. G., A. V. Timohov, V. V. Fedorov (1986). Kurs metodov optimizatsii. Moskva: Nauka, s. 138. 